\title{
Smoothness of correlations in the Anderson model at strong disorder
}

\author{
Jean V. Bellissard ${ }^{1 *}$ Peter D. Hislop ${ }^{2 \dagger}$ \\ ${ }^{1}$ Georgia Institute of Technology, School of Mathematics, Atlanta, GA 30332-0160 \\ ${ }^{2}$ Department of Mathematics, University of Kentucky, Lexington, KY 40506-0027 \\ July 4, 2018
}

\begin{abstract}
We study the higher-order correlation functions of covariant families of observables associated with random Schrödinger operators on the lattice in the strong disorder regime. We prove that if the distribution of the random variables has a density analytic in a strip about the real axis, then these correlation functions are analytic functions of the energy outside of the planes corresponding to coincident energies. In particular, this implies the analyticity of the density of states, and of the current-current correlation function outside of the diagonal. Consequently, this proves that the current-current correlation function has an analytic density outside of the diagonal at strong disorder.
\end{abstract}

\section{Contents}

1 Correlation Functions 2

1.1 Contents of the Paper . . . . . . . . . . . . . . . . . . . . 4

2 The Models, Hvpotheses, and the Main Result 5

3 Random Walk Expansion of the Resolvent 4

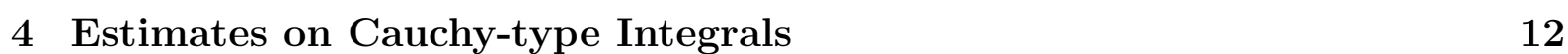

5 Analyticity of the Density of States 15

$\begin{array}{lll}6 & \text { Estimates on } N \text {-point Cauchy-type Integrals } & 17\end{array}$

*Partially supported by NSF Grant 0300398 .

${ }^{\dagger}$ Partially supported by NSF Grant 0503784 
KEY WORDS: random operators, transport, correlations

\section{Correlation Functions}

The study of the higher-order correlation functions for random Schrödinger operators is essential for an understanding of the transport properties of the system. In this paper, we study the correlation functions for covariant families of observables associated with random Anderson-type Schrödinger operators on $d$-dimensional lattices $\mathbb{Z}^{d}$ in the strong disorder regime. The Anderson model is given by the following random Hamiltonian acting on $\ell^{2}\left(\mathbb{Z}^{d}\right)$

$$
H_{\omega} \psi(x)=\lambda \sum_{y ;|y-x|=1} \psi(y)+V_{\omega}(x) \psi(x), \quad \text { for } \psi \in \ell^{2}\left(\mathbb{Z}^{d}\right) .
$$

Here $\lambda$ is a small real parameter providing a measure of the disorder of the system. The random potential $V_{\omega}$ is determined by a family of independent, identically distributed (iid) random variables $\omega=\left(V_{\omega}(x)\right)_{x \in \mathbb{Z}^{d}}$ with a common distribution given by a density $g(V) d V$. We assume that the density $g$ is analytic in a strip about the real axis. We write $\mathbb{P}$ for the probability measure generated by $g$ as an infinite product measure on the probability space $\Omega$. Precise assumptions and formulation of the results are given in the next section.

In order to describe our results, let us suppose that we have a family of covariant observables $A_{\omega, i}$, for $i=1, \ldots, N$. A basic example is the $j^{\text {th }}$-component of the velocity operator $V_{j}=i\left[H_{\omega}, x_{j}\right]$, that is independent of $\omega$. The resolvent $R_{\omega}(z)=\left(H_{\omega}-z\right)^{-1}$ for $H_{\omega}$ has matrix elements $G_{\omega}(x, y ; z)=\left\langle x\left|R_{\omega}(z)\right| y\right\rangle$ giving the Green function. The associated spectral density operator $\rho_{\omega}(E)$ is given by $\lim _{\epsilon \rightarrow 0} \Im\left(H_{\omega}-E-i \epsilon\right)^{-1}$. The $N-$ point correlation function $K_{N}$, associated with a covariant family $\left\{A_{\omega, j} \mid j=1, \ldots, N\right\}$, is given by

$$
K_{N}\left(E_{1}, E_{2}, \ldots, E_{N}\right) \equiv \int_{\Omega} d \mathbb{P}(\omega)\left\langle 0\left|\rho_{\omega}\left(E_{1}\right) A_{\omega, 1} \rho_{\omega}\left(E_{2}\right) A_{\omega, 2} \cdots \rho_{\omega}\left(E_{N}\right) A_{\omega, N}\right| 0\right\rangle
$$

We are interested in the behavior of $K_{N}$ as a function of the energies $E_{j}, j=1, \ldots, N$. It is not a priori clear that $K_{N}$ in (2) is well-defined. We will prove, in fact, that $K_{N}\left(E_{1}, \ldots, E_{N}\right)$ is real analytic in a region of $\mathbb{R}^{N}$ away from the planes where $E_{j}=E_{i}$, for $i \neq j$. 
As defined in (2), the first correlation function $K_{1}(E)$, traditionally noted as $\rho(E)$, is the density of states (DOS) of the family $H_{\omega}$. This function has been extensively studied, especially in one-dimension, and we refer the reader to the monographs [8, 17] for results on the regularity of the DOS for one-dimensional lattice models. In this article, we are especially concerned with lattice models in $d \geq 2$ for which there are very few analyticity results. Constantinescu, Fröhlich, and Spencer [10] studied the DOS for lattice models using the random walk expansion described in section 3. First, these authors proved two results independent of the (nonzero) disorder. They proved that if $g$ is analytic in a strip of width $\delta>4 d$, then $\rho(E)$ is analytic in a small strip around $|\Re E|>>1$. They also proved that if $g$ is Gaussian, then $\rho(E)$ is analytic for $|\Re E|>>1$, in a region $|\Im E|<(1 / \sqrt{2})|\Re E|$. Furthermore, the DOS decays like a Gaussian as $|E| \rightarrow \infty$ along the real axis. Note that both of these results are large energy results. Secondly, they proved that for $g$ Gaussian and large disorder, the DOS $\rho(E)$ is analytic in a neighborhood of the real axis $\mathbb{R}$. Later, Bovier, Campanino, Klein, and Perez [7] obtained stronger smoothness results on the DOS using a supersymmetric representation of the Green's function. These authors (actually, attributed to Klein and Perez, unpublished, in [7] ) prove two classes of results of interest here. First, they prove that if the characteristic function $h$ of the probability measure satisfies $(1+t)^{(d+k)} h(t) \in L^{1}(\mathbb{R})$, then $N(E) \in C^{(k+1)}(\mathbb{R})$, for all disorder sufficiently large (including $k=\infty$ ). Secondly, if $e^{\alpha t} h(t)$ is bounded for some $\alpha>0$, then for any $0<\alpha_{1}<\alpha$, there is a constant $\lambda_{1}>0$ so that for any $0 \leq \lambda<\lambda_{1}$, the IDS $N(E)$ is analytic in a strip $|\Im E|<\alpha_{1}$. Our results, restricted to the case $N=1$, are essentially the same as this last mentioned result on the analyticity of the DOS in a strip about the real axis provided the density has a continuation. Our method of proof is completely different and generalizes to any higher-order correlation functions.

The higher-order correlation functions have not been studied systematically, although L. Pastur [16], and one of the authors [3, 4], have long emphasized their importance in transport problems. The second-order correlation functions $K_{2}\left(E_{1}, E_{2}\right)$, for the choices $A_{\omega, i}=V_{i}$, are called the current-current correlation functions. These functions (actually measures) play an important role in the theory of conductivity. Until now, it was not known if the measure has a density. Our result on the current-current correlation function is the first proving the existence of a density for this measure away from the diagonal. We will comment further on this in section 2. The second-order correlation function has been studied by Pastur and Figotin for a one-dimensional quasi-periodic Schrödinger operator [11. The strong disorder expansion of the second moment suggested by Pastur was developed in Kirsch, Lenoble, Pastur [14] providing insight into the behavior of the function. In general, very little is known about this function and one of our results is that this function is analytic away from the diagonal $E_{1}=E_{2}$. The DC conductivity requires control of the two-point correlation function on the diagonal $E_{1}=E_{2}$. Although we do not achieve this result in this paper, we give the first results in the study of the correlation functions.

The higher-order correlation functions $K_{N}$ correspond to not-necessarily-positive, bounded, 
Radon measures $K_{N}\left(d E_{1}, \ldots, d E_{N}\right)$. Indeed, for any $N$, if $f_{1}, \cdots, f_{N}$ are smooth functions with compact support on $\mathbb{R}$ then

$\mathcal{T}_{\mathbb{P}}\left\{\nabla_{j_{1}} H f_{1}(H) \nabla_{j_{2}} H f_{2}(H) \cdots \nabla_{j_{N}} H f_{N}(H)\right\}=\int_{\mathbb{R}^{N}} K_{N}\left(d E_{1}, \ldots, d E_{N}\right) f_{1}\left(E_{1}\right) \cdots f_{N}\left(E_{N}\right)$,

where $j_{k} \in\{1, \ldots, d\}$. The left-hand side of (3) defines tempered distribution in $N$ variables. Moreover, the left-hand side is multilinear and bounded with respect to the sup norm of the $f_{i}$ 's since the operator $\nabla_{j} H$ is bounded for lattice models. Therefore $K_{N}$ belongs to the dual space of $\mathcal{C}_{0}(\mathbb{R})^{\otimes N} \simeq \mathcal{C}_{0}\left(\mathbb{R}^{N}\right)$, namely it is a bounded Radon measure on $\mathbb{R}^{N}$. Except for the cases $N=1$ and $N=2$, corresponding to the density of states and the current-current correlation function, respectively, this measure is not necessarily positive.

Control of higher-order correlation functions $K_{N}$ seems to be necessary, for example, in order to control growth in a dissipative model describing the Mott variable-range hopping. The higher-order moments of the position operator $R_{j}$ are also controlled by the higherorder correlation functions. To see this, we note that

$$
R_{j}(t)-R_{j}(0)=-i \int_{0}^{t} d s U_{s}\left[H, R_{j}\right] U_{s}^{*}=-i \int_{0}^{t} d s U_{s} \nabla_{j} H U_{s}^{*},
$$

where $U_{s}=e^{-i s H}$ and $\nabla_{j} H=-i\left[H, R_{j}\right]$ (see (6)). Consequently, there is a function $F_{t}\left(E_{1}, \ldots, E_{2 n}\right)$, depending on $t$, so that the $(2 n)^{t h}$-moment of the position operator $R_{j}(t)-R_{j}(0)$, localized to an energy interval $\Delta$ by projector $P_{\Delta}$, may be written as

$$
\mathcal{T}_{\mathbb{P}}\left(P_{\Delta}\left|R_{j}(t)-R_{j}(0)\right|^{2 n} P_{\Delta}\right)=\int_{\mathbb{R}^{2(n-1)} \times \Delta} K_{2 n}\left(d E_{1}, \ldots, d E_{2 n}\right) F_{t}\left(E_{1}, \ldots, E_{2 n}\right),
$$

where

$$
F_{t}\left(E_{1}, \ldots, E_{2 n}\right)=\prod_{j=1}^{2 n} \frac{\sin \left[(t / 2)\left(E_{j}-E_{j-1}\right)\right]}{E_{j}-E_{j-1}} .
$$

Our results on the current-current correlation functions, and the higher-order correlation functions, are the first results proving the smoothness of these correlation functions outside of the coincident planes.

\subsection{Contents of the Paper}

We state the main hypotheses on the models and the main results in section 2 . We introduce the random walk expansion of the resolvent in section 3. In section 4, we give some basic estimates on Cauchy-type integrals. To illustrate our method, we apply these to prove the analyticity of the DOS in section 5. We extend these estimates to multiple Cauchy-type integrals in section 6 . Analyticity of the boundary values of the Cauchytype integrals is proved in section 7 . The main result on the analyticity of the correlation functions is proved in sections 8 . 


\section{The Models, Hypotheses, and the Main Result}

We now provide precise hypotheses and formulation of our results. Throughout this paper, we will assume that the single-site probability density $g$ satisfies the following properties:

1. Analyticity: $g$ can be continued as a holomorphic function in the strip $\mathcal{B}_{r}=\{z \in$ $\mathbb{C} ;|\Im z|<r\}$

2. Boundedness: $\|g\|_{r}:=\sup _{|w|<r} \int_{\mathbb{R}} d v|g(v+\imath w)|<\infty$.

The space of functions fulfilling these conditions will be denoted by $\mathcal{H}_{r}$ and it will be endowed with the norm $\|\cdot\|_{r}$. Note that if $0<r^{\prime} \leq r$, then the norm satisfies $\|g\|_{r^{\prime}} \leq\|g\|_{r}$, for $g \in \mathcal{H}_{r}$. In addition, it is required that $g$ define a probability distribution with zero mean and a finite second moment, namely $g(v) \geq 0$, for $v \in \mathbb{R}$, and

$$
\int_{\mathbb{R}} d v g(v)=1, \quad \int_{\mathbb{R}} d v g(v) v=0, \quad \int_{\mathbb{R}} d v g(v) v^{2} \equiv M_{g}<\infty .
$$

The measure space in which $\omega$ lives will be denoted by $\Omega$ and the probability measure defined by the infinite product of $g(V) d V$ will be denoted $\mathbb{P}$. Obviously $\mathbb{Z}^{d}$ acts on $\Omega$ by bimeasurable bijective maps and $\mathbb{P}$ is $\mathbb{Z}^{d}$-invariant and ergodic. A covariant operator is a family $A=\left(A_{\omega}\right)_{\omega \in \Omega}$ of bounded operators on $\mathfrak{H}=\ell^{2}\left(\mathbb{Z}^{d}\right)$ such that

1. The map $\omega \in \Omega \mapsto A_{\omega} \in \mathcal{B}(\mathfrak{H})$ is strongly $\mathbb{P}$-measurable ${ }^{1}$.

2. If $T(a)$ denotes the unitary operator of translation by $a$ then $T(a) A_{\omega} T(a)^{-1}=A_{\mathrm{T}^{a} \omega}$, if $\mathrm{T}^{a}$ is the action of $a \in \mathbb{Z}^{d}$ on $\Omega$.

The Anderson Hamiltonian $H_{\omega}$, defined in (11), is an example of a family of covariant operators whenever the distribution of the random variables $V_{\omega}(x)$ has compact support. However, due to the analyticity condition on $g$, the Hamiltonians $H_{\omega}$ are almost surely unbounded and selfadjoint. It is required then that the field of resolvents $\omega \in \Omega \mapsto$ $\left(z \mathbf{1}-H_{\omega}\right)^{-1} \in \mathcal{B}(\mathfrak{H})$ be measurable. The set of covariant operators is a von Neumann algebra $^{2}$ with the pointwise algebraic operations (sum, product, adjoint) and with the

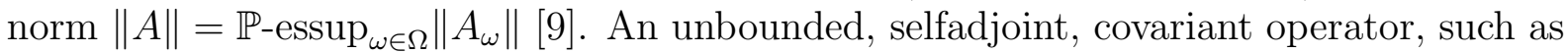
$H_{\omega}$, is said to be affiliated to this algebra if all its spectral projections for bounded Borel subsets of $\mathbb{R}$ belong to this algebra. There is a canonical trace on this algebra given by [5, 9]

$$
\mathcal{T}_{\mathbb{P}}(A)=\int_{\Omega} d \mathbb{P}(\omega)\left\langle 0\left|A_{\omega}\right| 0\right\rangle=\lim _{\Lambda \uparrow \mathbb{Z}^{d}} \frac{1}{|\Lambda|} \operatorname{Tr}_{\Lambda}\left(A_{\omega}\right), \quad \mathbb{P} \text { a.e. }
$$

\footnotetext{
${ }^{1}$ Note that the Borel structure of $\mathcal{B}(\mathfrak{H})$ is the same for the weak and strong topologies since $\mathfrak{H}$ is separable.

${ }^{2} \mathrm{~A}$ von Neumann algebra is a $C^{*}$-algebra with a predual [18].
} 
This von Neumann algebra is denoted by $L^{\infty}\left(\mathcal{T}_{\mathbb{P}}\right)^{3}$. Let $\vec{R}=\left(R_{1}, \cdots, R_{d}\right)$ be the position operator acting on $\mathfrak{H}$ as a multiplication operator. Then a derivation $\vec{\nabla}$ on the algebra is defined by

$$
\vec{\nabla}=\left(\partial_{1}, \cdots, \partial_{d}\right), \quad \text { with }\left(\partial_{\mu} A\right)_{\omega}=\imath\left[R_{\mu}, A_{\omega}\right],
$$

on the dense subalgebra of covariant operators so that the operators $\left(\partial_{\mu} A\right)_{\omega}$ are bounded almost surely. The Integrated Density of States (IDS) has been proved to satisfy the Shubin-Pastur formula (see [20, 17, 1, 2])

$$
\mathcal{N}(E)=\lim _{\Lambda \uparrow \mathbb{Z}^{d}} \frac{1}{|\Lambda|} \#\left\{\text { eigenvalues of } H_{\omega} \Upsilon_{\Lambda} \leq E\right\}=\mathcal{T}_{\mathbb{P}}(\chi(H \leq E)) \quad \text { a.e. } \omega,
$$

where $\chi(H \leq E)$ denotes the spectral projection of the covariant Hamiltonian $H_{\omega}$ on the interval $(-\infty, E]$. The density of states (DOS) is the Lebesgue-Stieljes measure $d \mathcal{N}$.

Transport properties are expressed through the current-current correlation function defined as the positive matrix valued measure on $\mathbb{R}^{2}$ given by

$$
\mathcal{T}_{\mathbb{P}}\left(f(H) \partial_{\nu} H g(H) \partial_{\nu^{\prime}} H\right)=\int_{\mathbb{R} \times \mathbb{R}} m_{\nu, \nu^{\prime}}\left(d E, d E^{\prime}\right) f(E) g\left(E^{\prime}\right),
$$

for $f, g \in \mathcal{C}_{0}(\mathbb{R})$. Since the electric current operator is given by $\vec{J}=(e / \hbar) \vec{\nabla} H$, the measure $m$ is formally given by $m_{\nu, \nu^{\prime}}\left(d E, d E^{\prime}\right)=\left\langle E\left|J_{\nu}\right| E^{\prime}\right\rangle\left\langle E^{\prime}\left|J_{\nu^{\prime}}\right| E\right\rangle d E d E^{\prime}$ (up to a multiplicative constant) in terms of the matrix elements of the current in the eigenbasis of the Hamiltonian.

One of the main problems for transport in such system is to show that this measure is absolutely continuous with smooth density. We prove a first result in this direction. Namely, we prove that the density exists and is analytic outside of the diagonal at all energies in the strong disorder regime. More precisely, let $\underline{A}=\left\{A_{1}, \ldots, A_{N}\right\}$ be a finite family of covariant operators as defined above, such as the velocity operators. The $N$-point Green function associated with $\underline{A}$ is defined by

$$
G_{\underline{A}}(\underline{z})=\mathcal{T}_{\mathbb{P}}\left(\left(H-z_{1}\right)^{-1} A_{1} \cdots\left(H-z_{N}\right)^{-1} A_{N}\right), \quad \underline{z}=\left(z_{1}, \cdots, z_{N}\right) \in(\mathbb{C} \backslash \mathbb{R})^{N} .
$$

Note that the spectral function is a linear combination of two Green functions with complex-conjugate imaginary parts. The DOS is recovered by taking $N=1, A_{1}=1$, and taking the imaginary part. The two-point correlation function is recovered by taking $N=2, A_{1}=A_{2}=\partial_{\nu} H$, expressing the spectral family in terms of the Green's functions via Stone's formula, and by considering the discontinuity of $G_{\underline{A}}(\underline{z})$ along $z_{i}=E_{i} \in \mathbb{R}$. The discontinuities are expressed in terms of the boundary values defined as follows: let

\footnotetext{
${ }^{3}$ For an intrinsic definition without the help of $\mathfrak{H}$, see [2, 9]
} 
$\underline{\sigma}=\left(\sigma_{1}, \cdots, \sigma_{N}\right) \in\{+,-\}^{N}$, and let $\underline{\epsilon}=\left(\epsilon_{1}, \cdots, \epsilon_{N}\right) \in \mathbb{R}_{+}^{N}$. Then, with the notation $\underline{\sigma} \cdot \underline{\epsilon}=\left(\sigma_{1} \epsilon_{1}, \cdots, \sigma_{N} \epsilon_{N}\right)$, we define the boundary values of (요) as

$$
G \underline{\underline{A}}(\underline{E})=\lim _{\epsilon \downarrow 0} G_{\underline{A}}(\underline{E}+\imath \underline{\sigma} \cdot \underline{\epsilon}) .
$$

The $N$-point correlation functions $K_{N}$ defined in (2) can be expressed as a linear combination of these boundary values. Our main result is the following theorem.

Theorem 1 Let $H_{\omega}$ be the Anderson Hamiltonian defined in (1) with a distribution $g \in$ $\mathcal{H}_{r}$. Then, given $N \in \mathbb{N}$, there is $a_{0} \equiv a_{0}(r, g, d, N)>0$ such that any family $\underline{A}=$ $\left\{A_{1}, \ldots, A_{N}\right\}$ of covariant operators, the boundary values of the $N$-point Green function $G_{\underline{A}}$ defined in (8) are real analytic in domains $\left\{\underline{E} \in \mathbb{R}^{N} ;\left|E_{i}-E_{j}\right|>a_{0}|\lambda|\right\}$.

The proof will use the random walk expansion proposed by Fröhlich and Spencer [12] and used by Constantinescu, Fröhlich, and Spencer [10] for the DOS, which applies in the perturbative domain $|\lambda| \ll 1$ of strong disorder. As an immediate corollary we recover the analyticity result of Bovier, Campanino, Klein, and Perez [7], improving the result of Constantinescu, Fröhlich, and Spencer [10], for the case $N=1$. More importantly, we obtain the first results on the existence of a density for the current-current correlation function.

Corollary 1 For the Anderson model, under the assumption of Theorem 1, for any $\epsilon>0$, there exists a $\lambda_{r, \epsilon}>0$ so that for $|\lambda|<\lambda_{r, \epsilon}$, the DOS is analytic in a strip of width $r-\epsilon>0$ about the real axis. Furthermore, there is a constant $0<a_{2}<\infty$ so that the currentcurrent correlation functions are real analytic in $\left(E, E^{\prime}\right)$ on $\mathbb{R}^{2} \backslash\left\{\left(E, E^{\prime}\right)|| E-E^{\prime} \mid \leq\right.$ $\left.a_{2}|\lambda|\right\}$.

It is not expected that the current-current correlation function $m\left(E, E^{\prime}\right)$ be analytic near the diagonal. In fact, a perturbative approach [14, 16] predicts $m\left(E_{1}, E_{2}\right) \simeq C \mid E_{1}-$ $\left.E_{2}\right|^{2} \ln ^{d+1}\left|E_{1}-E_{2}\right|$, and a recent result by Klein, Lenoble, and Müller [13] proved an upper bound on the AC conductivity in the spirit of Mott's formula. This has the form $\bar{\sigma}_{E}(\nu) \leq C_{0} \nu^{2} \log \left(\frac{1}{\nu}\right)$, as $\nu \rightarrow 0$, for energies $E$ in the localization regime at strong disorder. The averaged conductivity $\bar{\sigma}_{E}(\nu)$ is defined as $\nu^{-1} \Sigma_{E}([0, \nu])$ for a well-defined conductivity measure $\Sigma_{E}$ (see [13]). If the current-current correlation function has a density $m\left(E_{1}, E_{2}\right)$ near the diagonal $E_{1} \approx E_{2}$, then this result implies $m\left(E_{1}, E_{2}\right) \leq C\left|E_{1}-E_{2}\right|^{2} \ln ^{d+2}\left|E_{1}-E_{2}\right|$ in the localization regime at strong disorder. The assumption that such a density exists near the diagonal is still unproven. We also have reason to believe that this upper bound might be supplemented by a similar lower bound, namely

Problem 1 Prove that the current-current correlation function is given by a density that vanishes at $E_{1}=E_{2}$ like $\left|E_{1}-E_{2}\right|^{2} \ln ^{\alpha}\left|E_{1}-E_{2}\right|$, for some constant $\alpha>0$ depending on the dimension $d$. 
We mention that this behavior is not consistent with the behavior predicted by the Mott variable range hopping conductivity argument [15, 19, which should, in principle, imply the existence of an essential singularity at coincident energies. If so, it casts some doubt on the ability of the one-particle Anderson model to account for the properties of semiconductors at very low temperatures. We intend to discuss this behavior for the 2-point, and the general $N$-point correlation functions, in the strong localization regime in a companion article [6].

\section{Random Walk Expansion of the Resolvent}

The method used in these notes is the one proposed in the early days of the Anderson model by Fröhlich and Spencer [12] and used to treat the DOS for Gaussian distributions by Constantinescu, Fröhlich, and Spencer [10]. It is a simple perturbation expansion in the small parameter $\lambda$ appearing in the definition (11). From the definition of $G_{\underline{A}}(\underline{z})$ in (81), it follows that

$$
G_{\underline{A}}(\underline{z})=\int_{\Omega} d \mathbb{P}(\omega)\left\langle 0\left|\frac{1}{H_{\omega}-z_{1}}\right| x_{1}\right\rangle\left\langle x_{1}\left|A_{1, \omega}\right| y_{1}\right\rangle \cdots\left\langle y_{N-1}\left|\frac{1}{H_{\omega}-z_{N}}\right| x_{N}\right\rangle\left\langle x_{N}\left|A_{N, \omega}\right| 0\right\rangle,
$$

where repeated coordinates are summed over all $\mathbb{Z}^{d}$. We first show how a general $N$ point function $G_{\underline{A}}(\underline{z})$ can be approximated by an $N$-point function constructed from simpler covariant operators $A$ that we call $r$-monomials. These are effectively finite-range operators with analytic coefficients.

Definition 1 Given $r>0$, an element $A \in L^{\infty}\left(\mathcal{T}_{\mathbb{P}}\right)$ is called an $r$-monomial if there is a family $b_{1}, \cdots, b_{L}$ of bounded holomorphic complex-valued functions in the strip $\mathcal{B}_{r}=\{z \in$ $\mathbb{C} ;|\Im z|<r\}$, vanishing at infinity, and a finite set of points $u_{1}, \cdots, u_{L} \in \mathbb{Z}^{d}$, such that the matrix elements of $A_{\omega}$ satisfy

$$
\left\langle 0\left|A_{\omega}\right| x\right\rangle=\prod_{j=1}^{L} b_{j}\left(V\left(u_{j}\right)\right) \delta_{x, u_{0}} .
$$

A covariant operator $A$ is an $r$-polynomial if it is a finite sum of $r$-monomials.

Examples of covariant operators $A_{\omega}$ include the Laplacian $A=\Delta$, the velocity operators $V_{j}=i\left[H_{\omega}, x_{j}\right]$, and inverses of even polynomials in the random potential $A=V_{\omega}$ with positive coefficients. These are not $r$-monomials since the coefficients don't vanish at infinity. However, they can be well-approximated by finite linear combinations of $r$ monomials as the next proposition shows. We call a covariant operator $A_{\omega}$ a finite-range covariant operator if there is a finite number $R>0$ such that if $x, y \in \mathbb{Z}^{d}$ with $|x-y| \geq R$, then $\left\langle x\left|A_{\omega}\right| y\right\rangle=0$ almost surely. 
Proposition 1 Given a family $\underline{A}$ of $N$ elements in $L^{\infty}\left(\mathcal{T}_{\mathbb{P}}\right)$, the $N$-point function $G_{\underline{A}}$ can be approximated uniformly on any compact subset of $(\mathbb{C} \backslash \mathbb{R})^{N}$ by a sequence of linear combinations of $N$-point functions involving only $r$-monomials.

The proof involves the following steps.

Lemma 1 Let $A$ be an element of the $L^{\infty}\left(\mathcal{T}_{\mathbb{P}}\right)$. Then,

(i) its matrix elements can be written as $\left\langle x\left|A_{\omega}\right| y\right\rangle=a\left(\mathrm{~T}^{-x} \omega, y-x\right)$ where, for each $u \in \mathbb{Z}^{d}$, the map $a_{u}: \omega \in \Omega \mapsto a(\omega, u)$ belongs to $L^{\infty}(\Omega, \mathbb{P})$.

(ii) the sum $\sum_{u}\left|a_{u}\right|^{2}$ converges in $L^{\infty}(\Omega, \mathbb{P})$.

Proof: The covariance condition applied to $A$ implies $\left\langle x\left|A_{\omega}\right| y\right\rangle=\left\langle x-s\left|A_{\mathrm{T}^{-s} \omega}\right| y-s\right\rangle$ $\mathbb{P}$-almost surely for all $s \in \mathbb{Z}^{d}$. If we define $a_{u}(\omega)=a(\omega, u) \equiv\left\langle 0\left|A_{\omega}\right| u\right\rangle$, then choosing $s=x$ leads to $\left\langle x\left|A_{\omega}\right| y\right\rangle=a\left(\mathrm{~T}^{-x} \omega, y-x\right)$. Since $\omega \in \Omega \mapsto A_{\omega}$ is measurable, so are each of the maps $a_{u}$. Since $A$ is bounded, it follows that $\left\|a_{u}\right\|_{L^{\infty}} \leq\|A\|$. Hence $a_{u} \in L^{\infty}(\Omega, \mathbb{P})$. In the same way, one shows that $\left\langle 0\left|A_{\omega} A_{\omega}^{*}\right| 0\right\rangle=\sum_{u \in \mathbb{Z}^{d}}\left|a_{u}(\omega)\right|^{2} \leq\|A\|^{2}$, showing that this sum actually converges in $L^{\infty}(\Omega, \mathbb{P})$.

Lemma 2 Any element $A \in L^{\infty}\left(\mathcal{T}_{\mathbb{P}}\right)$ can be weakly approximated by a sequence of $r$ polynomials.

Proof: Thanks to Lemma 1(ii), $A$ can be uniformly approximated by a finite range operator, namely given $\epsilon>0$, there is $N \in \mathbb{N}$ such that $\sum_{|u|>N}\left|a_{u}\right|^{2} \leq \epsilon^{2}$. Hence setting $a_{u}^{(N)}=a_{u}$, if $|u| \leq N$, and $a_{u}^{(N)}=0$, otherwise, we obtain a finite range operator $A_{N} \in L^{\infty}\left(\mathcal{T}_{\mathbb{P}}\right)$ such that $\left\|A-A_{N}\right\| \leq \epsilon$. On the other hand, since $\Omega$ can be taken as the Cartesian product $\mathbb{R}^{\mathbb{Z}^{d}}$, any element of $L^{\infty}(\Omega, \mathbb{P})$ can be weakly approximated (for the weak-* topology) by a continuous cylindrical function. A function $f: \Omega \mapsto \mathbb{C}$ is called cylindrical if there is $L \in \mathbb{N}_{*}$, a continuous function $F \in \mathcal{C}_{0}\left(\mathbb{R}^{L}\right)$, vanishing at infinity, and a finite subset $\left\{x_{1}, \cdots, x_{L}\right\} \subset \mathbb{Z}^{d}$ such that $f(\omega)=F\left(V_{\omega}\left(x_{1}\right), \cdots, V_{\omega}\left(x_{L}\right)\right)$. Since $\mathcal{C}_{0}\left(\mathbb{R}^{L}\right)$ is the uniform closure of the algebraic tensor product $\mathcal{C}_{0}(\mathbb{R})^{\otimes L}$ such a function $F$ can be uniformly approximated by a finite sum of functions of the form $f_{1}\left(V_{\omega}\left(x_{1}\right)\right) \cdots f_{L}\left(V_{\omega}\left(x_{L}\right)\right)$, where $f_{i} \in \mathcal{C}_{0}(\mathbb{R})$. At last, the space $\mathcal{H}_{r}$, made of functions in $\mathcal{C}_{0}(\mathbb{R})$ that can be continued as holomorphic functions on the strip $\mathcal{B}_{r}$ vanishing at infinity, is dense in $\mathcal{C}_{0}(\mathbb{R})$.

We let $\mathcal{H}$ be the space of functions on $(\mathbb{C} \backslash \mathbb{R})^{N}$ that are holomorphic and bounded at infinity, and endowed with the topology of uniform convergence on compact sets.

Lemma 3 The map $\underline{A}=\left(A_{1}, \cdots, A_{N}\right) \in L^{\infty}\left(\mathcal{T}_{\mathbb{P}}\right)^{\times N} \mapsto G_{\underline{A}} \in \mathcal{H}$ is multilinear and weak-* continuous.

Proof: This is a consequence of the GNS-representation theorem and the property that on a von Neumann algebra the weak and the strong topology coincide on bounded sets. 
Proof of Proposition 1: It is a consequence of the previous Lemmas 11, 2] and 3.

We now return to the expansion (9). Since the kinetic term in (11) is given by the discrete Laplacian (without diagonal term), the Green function can then be expanded in formal power series in $\lambda$. This gives

$$
\left\langle y\left|\frac{1}{H_{\omega}-z}\right| x\right\rangle=\sum_{j=0}^{\infty}(-\lambda)^{j}\left\langle y\left|\frac{1}{V-z}\left[H_{0} \frac{1}{V-z}\right]^{j}\right| x\right\rangle .
$$

We next use the fact that the Laplacian $H_{0}$ couples only nearest-neighbor terms to obtain a path expansion of matrix elements of the resolvent. We need some notation. We denote by $\gamma$ a path from $y$ to $x$, namely it is a sequence $\left(x_{0}=y, x_{1}, \cdots, x_{n-1}, x_{n}=x\right)$ where $x_{k} \in \mathbb{Z}^{d}$ for all $k$ 's and $\left|x_{k}-x_{k-1}\right|=1$, for $1 \leq k \leq n$. Note that all the points need not be distinct. The points $x_{0}$ and $x_{n}$ are called the initial and the final points of $\gamma$ and will be denoted by $\partial_{0} \gamma$ and $\partial_{1} \gamma$, respectively. We call $n=|\gamma|$ the length of $\gamma$. We denote by $\mathcal{V}(\gamma)=\left\{x_{k} ; 0 \leq k \leq n\right\}$ the family of (distinct) vertices of $\gamma^{4}$. It will be convenient to denote by $\# \gamma$ the cardinality of $\mathcal{V}(\gamma)$ so that $\# \gamma \leq n+1$. The path expansion takes the form:

$$
\left\langle y\left|\frac{1}{H_{\omega}-z}\right| x\right\rangle=\sum_{\gamma: y \mapsto x}(-\lambda)^{|\gamma|} \prod_{k=0}^{n} \frac{1}{V\left(x_{k}\right)-z} .
$$

Given $u \in \mathbb{Z}^{d}$, let $n_{\gamma}(u)$ be the number of $k \in[0, n]$ such that $x_{k}=u$, so that $n+1=$ $|\gamma|+1=\sum_{u \in \mathbb{Z}^{d}} n_{\gamma}(u)$. Hence (10) can be written as

$$
\left\langle y\left|\frac{1}{H_{\omega}-z}\right| x\right\rangle=\sum_{\gamma: y \mapsto x}(-\lambda)^{|\gamma|} \prod_{u \in \mathbb{Z}^{d}} \frac{1}{(V(u)-z)^{n_{\gamma}(u)}} .
$$

This formula will be used to expand $G_{\underline{A}}(\underline{z})$ given in (91). Since $G_{\underline{A}}(\underline{z})$ contains $N$ such Green functions, this expansion will require $N$ paths, namely a family $\Gamma$ of $N$ paths, $\Gamma=\left(\gamma_{1}, \cdots, \gamma_{N}\right)$. For such a family, the following notation will be used: $|\Gamma|=\sum_{i=1}^{N}\left|\gamma_{i}\right|$, $\mathcal{V}(\Gamma)=\cup_{i=1}^{N} \mathcal{V}\left(\gamma_{i}\right), \# \Gamma$ is the cardinality of $\mathcal{V}(\Gamma)$, while if $u \in \mathbb{Z}^{d}, n_{\Gamma}(u)=\sum_{i=1}^{N} n_{\gamma_{i}}(u)$. The initial and final points of $\Gamma$ are defined by $\partial_{0} \Gamma=\partial_{0} \gamma_{1}$ and $\partial_{1} \Gamma=\partial_{1} \gamma_{N}$, respectively. An $N$-path is $\underline{A}$ compatible if $\left\langle\partial_{1} \gamma_{i}\left|A_{i, \omega}\right| \partial_{0} \gamma_{i+1}\right\rangle \neq 0$, for $i \in[1, N]$, with the convention $\gamma_{N+1}=\gamma_{1}$. Let then $\mathcal{P}(\underline{A})$ be the set of $\underline{A}$-compatible $N$-paths with $\partial_{0} \Gamma=\partial_{1} \Gamma=0$. Thanks to (11), the $N$-point correlation can be written as

$$
G_{\underline{A}}(\underline{z})=\int_{\Omega} d \mathbb{P}(\omega) \sum_{\Gamma \in \mathcal{P}(\underline{A})}(-\lambda)^{|\Gamma|} \prod_{u \in \mathbb{Z}^{d}} \prod_{i=1}^{N} \frac{1}{\left(V(u)-z_{i}\right)^{n_{\gamma_{i}}(u)}} \prod_{i=1}^{N}\left\langle\partial_{1} \gamma_{i}\left|A_{i, \omega}\right| \partial_{0} \gamma_{i+1}\right\rangle .
$$

\footnotetext{
${ }^{4}$ If the path $\gamma$ passes through the same vertex more than once, $\mathcal{V}(\gamma)$ has less than $n+1$ elements in general.
} 
Let the operators $A_{i, \omega}$ be all $r$-monomials. It follows from Definition 1 and Lemma 1 that their matrix elements factorize according to

$$
\left\langle x\left|A_{i, \omega}\right| y\right\rangle=\delta_{y-x, u_{i}} \prod_{u \in \mathbb{Z}^{d}} a_{i, u-x}(V(u)),
$$

for some $u_{i} \in \mathbb{Z}^{d}$ and where $a_{i, u}=1$ for all but a finite number of indices $(i, u)$. It follows that an $N$-path is $\underline{A}$-compatible if and only if

$$
\Gamma \in \mathcal{P}(\underline{A}) \Longleftrightarrow \partial_{0} \gamma_{i+1}-\partial_{1} \gamma_{i}=u_{i} \forall i \in[1, N] \text {. }
$$

Hence there is no need to insert the product of the Kronecker symbols associated with the matrix elements of the $A_{i, \omega}$ 's. Then, since the probability measure $\mathbb{P}$ factorizes with respect to the sites, the previous formula becomes:

$$
G_{\underline{A}}(\underline{z})=\sum_{\Gamma \in \mathcal{P}(\underline{A})}(-\lambda)^{|\Gamma|} \prod_{u \in \mathbb{Z}^{d}} \int_{-\infty}^{+\infty} d v g(v) \prod_{i=1}^{N} \frac{a_{i, u-\partial_{1} \gamma_{i}}(v)}{\left(v-z_{i}\right)^{n_{\gamma_{i}}(u)}} .
$$

As in (24) of Section [6, if $\underline{n}=\left(n_{1}, \cdots, n_{N}\right) \in \mathbb{N}^{N}$ and $\underline{z}=\left(z_{1}, \cdots, z_{N}\right) \in(\mathbb{C} \backslash \mathbb{R})^{N}$, it is convenient to set

$$
J_{\underline{n}}(h ; \underline{z})=\int_{-\infty}^{+\infty} d v h(v) \prod_{i=1}^{N} \frac{1}{\left(v-z_{i}\right)^{n_{i}+1}},
$$

Using the notation $\underline{n}_{\Gamma}(u)=\left(n_{\gamma_{1}}(u), \cdots, n_{\gamma_{N}}(u)\right)$ and $\underline{f}=(1,1, \cdots, 1)$, the previous formal $N$-path expansion becomes

$$
G_{\underline{A}}(\underline{z})=\sum_{\Gamma \in \mathcal{P}(\underline{A})}(-\lambda)^{|\Gamma|} \prod_{u \in \mathbb{Z}^{d}} J_{\underline{n}_{\Gamma}(u)-\underline{f}}\left(g_{\Gamma, u} ; \underline{z}\right)
$$

where

$$
g_{\Gamma, u}(v)=g(v) \prod_{i=1}^{N} a_{i, u-\partial_{1} \gamma_{i}}(v) .
$$

As a final result in this section, we estimate the number of $\underline{A}$-compatible $N$-paths of a given length.

Lemma 4 Let $\underline{A}$ be a family of $N$ r-monomials. Then the number of $\underline{A}$-compatible $N$ paths of total length $n$ is bounded from above by

$$
\#\{\Gamma \in \mathcal{P}(\underline{A}) ;|\Gamma|=n\} \leq(2 d)^{n} .
$$


Proof: The initial point of $\Gamma$ is fixed at $x=0$. There are $2 d$ ways of choosing a neighbor of 0 . This gives the first vertex $x_{1}$ of $\gamma_{1}$. If $x_{1}, x_{2}, \cdots, x_{j}$ have been chosen, there are again $2 d$ neighbors of $x_{j}$ giving $2 d$ admissible choices for $x_{j+1}$. Hence there are exactly $(2 d)^{\left|\gamma_{1}\right|}$ ways of choosing $\gamma_{1}$. Assume $\gamma_{1}, \cdots, \gamma_{k}$ have been chosen. Then, the final point $\partial_{1} \gamma_{k}$ is fixed so that the $\underline{A}$-compatibility fixes the initial point $\partial_{0} \gamma_{k+1}$ unambiguously due to (12). The same argument shows that the number of possible choices for $\gamma_{k+1}$ is at most $(2 d)^{\left|\gamma_{k+1}\right|}$. This leads to the result by recursion on $k \in[1, N]$.

\section{Estimates on Cauchy-type Integrals}

In order to prove analyticity of the correlation functions $G_{\underline{A}}(\underline{z})$, as expressed in (14), we first need to analyze the Cauchy-type integrals $J_{\underline{n}}(h ; \underline{z})$ defined in (13). We begin with estimates on the simplest form of these integrals for which $\underline{n}$ and $\underline{z}$ depend on one variable only. We will treat the general case in section 6 after we apply the results of this section to the DOS. The first result on the behavior of the density $g$ is the following

Lemma 5 Let $g \in \mathcal{H}_{r}$ and let $g^{(n)}$ be its $n^{\text {th }}$ derivative, then for any $0<\rho<r$, we have

$$
\sup _{z:|\Im z| \leq r-\rho}|g(z)| \leq \frac{1}{\pi \rho}\|g\|_{r}, \quad\left\|g^{(n)}\right\|_{r-\rho} \leq \frac{n !}{\rho^{n}}\|g\|_{r} .
$$

Proof: Let $z \in \mathcal{B}_{r-\rho}=\{z \in \mathbb{C}|| \Im z \mid<r-\rho\}$, and let $\gamma$ denote a path contained in the strip $\mathcal{B}_{r}$ homotopic to the circle centered at $z$ of radius $\rho$. Thanks to the Cauchy formula

$$
g(z)=\oint_{\gamma} \frac{d z^{\prime}}{2 \imath \pi} \frac{g\left(z^{\prime}\right)}{\left(z^{\prime}-z\right)}
$$

Taking $\gamma$ as the union of the lines $\gamma_{ \pm}=\{z+u \pm \imath \rho ; u \in \mathbb{R}\}$ this gives

$$
|g(z)|=\left|\int_{-\infty}^{+\infty} \frac{d u}{2 \imath \pi}\left\{\frac{g(u+z-\imath \rho)}{u-\imath \rho}-\frac{g(u+z+\imath \rho)}{u+\imath \rho}\right\}\right| \leq \frac{1}{\pi \rho}\|g\|_{r} .
$$

Using again the Cauchy formula, with now $\gamma$ being the circle centered at $z$ of radius $\rho$, gives

$$
g^{(n)}(z)=n ! \oint_{\gamma} \frac{d z^{\prime}}{2 \imath \pi} \frac{g\left(z^{\prime}\right)}{\left(z^{\prime}-z\right)^{n+1}}=\frac{n !}{\rho^{n}} \int_{0}^{2 \pi} \frac{d \theta}{2 \pi} g\left(z+\rho e^{\imath \theta}\right) e^{-\imath n \theta} .
$$

Integrating the absolute values of both sides over a line parallel to the real axis gives the result.

Let $I_{n}(g ; z)$ be defined by

$$
I_{n}(g ; z)=\int_{-\infty}^{+\infty} d v \frac{g(v)}{(v-z)^{n+1}}
$$


which is convergent for $\Im z \neq 0$. We have the following identities.

Lemma 6 If $g \in \mathcal{H}_{r}$, then for any $z \in \mathbb{C}$ with $\Im z \neq 0$,

$$
I_{n}(g ; z)=\frac{1}{n !} \frac{d^{n}}{d z^{n}} I_{0}(g ; z)=\frac{1}{n !} I_{0}\left(g^{(n)} ; z\right) .
$$

Proof: The first identity is a direct consequence of the definition. For the second equality, we note that

$$
\frac{d v}{(v-z)^{n+1}}=-\frac{1}{n} d \frac{1}{(v-z)^{n}} .
$$

Since $g \in \mathcal{H}_{r}$, it follows from an integration by parts that

$$
I_{n}(g ; z)=-\frac{1}{n} \frac{g(v)}{(v-z)^{n}} \uparrow_{-\infty}^{+\infty}+\frac{1}{n} \int_{-\infty}^{+\infty} d v \frac{g^{(1)}(v)}{(v-z)^{n}} .
$$

The first term vanishes while the second gives $I_{n}(g ; z)=I_{n-1}\left(g^{(1)} ; z\right) / n$. The formula follows by recursion.

Lemma 7 If $g \in \mathcal{H}_{r}$, then

$$
\lim _{\epsilon \downarrow 0} I_{0}(g, E \pm \imath \epsilon)=\int_{0}^{\infty} d u \frac{g(E+u)-g(E-u)}{u} \pm \imath \pi g(E) .
$$

Proof: Thanks to Cauchy's formula, this limit can be computed by using a deformed path $\gamma$ avoiding the point $z=E$. A possible choice, for positive imaginary part, is $\gamma=\gamma_{-} \cup \gamma_{0} \cup \gamma_{+}$, where $\gamma_{-}=(-\infty, \epsilon], \gamma_{0}=\left\{\epsilon e^{\imath \theta} ;-\pi \leq \theta \leq 0\right\}$, and $\gamma_{+}=[\epsilon,+\infty)$. This gives a decomposition of $I_{0}(g ; E+\imath 0)$ into three integrals $I_{-}+I_{0}+I_{+}$. After the change of variable $v=E \pm u$, the contributions of $\gamma_{ \pm}$are given by

$$
I_{-}=-\int_{\epsilon}^{\infty} \frac{d u}{u} g(E-u), \quad I_{+}=\int_{\epsilon}^{\infty} \frac{d u}{u} g(E+u) .
$$

Setting $v=E+\epsilon e^{\imath \theta}$ gives

$$
I_{0}=\int_{-\pi}^{0} \imath d \theta g\left(E+\epsilon e^{\imath \theta}\right) \stackrel{\epsilon \downarrow 0}{\longrightarrow} \imath \pi g(E) .
$$

Since $g(E+u)-g(E-u)$ vanishes like $\mathcal{O}(u)$ for $u \rightarrow 0$, the sum $I_{+}+I_{-}$converge as $\epsilon \rightarrow 0$ giving the result.

Lemma 8 For $r>0$, if $g \in \mathcal{H}_{r}$, then

$$
\sup _{E \in \mathbb{R}}\left|I_{0}(g ; E \pm \imath 0)\right| \leq\left(\left(\frac{8}{\pi}+2\right) \frac{1}{r^{2}}+\frac{1}{r}+1\right)\|g\|_{r} .
$$


Proof: Using the formula given in Lemma 17, the integral (16) over $u$ decomposes into $\int_{0}^{1} d u(\cdot)+\int_{1}^{\infty} d u(\cdot)$. For the first integral, we write $g(E+u)-g(E-u)=\int_{-u}^{u} g^{\prime}(E+x) d x$, and integrate by parts in the variable $u$, to obtain,

$$
\begin{aligned}
\int_{0}^{1} d u \frac{g(E+u)-g(E-u)}{u} & =\int_{0}^{1} \frac{d u}{u}\left[\int_{-u}^{+u} d x g^{(1)}(E+x)\right] \\
& =\int_{0}^{1} d x \ln (1 / x)\left(g^{(1)}(E+x)+g^{(1)}(E-x)\right) .
\end{aligned}
$$

Another integration by parts, gives

$$
\begin{aligned}
\int_{0}^{1} d u \frac{g(E+u)-g(E-u)}{u}= & \left(g^{(1)}(E+1)+g^{(1)}(E-1)\right) \\
& -\int_{0}^{1} d x\left(g^{(2)}(E+x)-g^{(2)}(E-x)\right)(x-x \ln x)(17)
\end{aligned}
$$

To bound this, we use Lemma 5, and obtain,

$$
\left|g^{(1)}\left(E^{\prime}\right)\right| \leq \frac{1}{\pi(r-\rho)}\left\|g^{(1)}\right\|_{r-\rho} \leq \frac{1}{\pi \rho(r-\rho)}\|g\|_{r}, \quad \forall E^{\prime} \in \mathbb{R}, \quad 0<\rho<r .
$$

The choice $\rho=r / 2$ gives the optimal bound on the right side, so that

$$
\sup _{E^{\prime} \in \mathbb{R}}\left|g^{(1)}\left(E^{\prime}\right)\right| \leq \frac{4}{\pi r^{2}}\|g\|_{r}
$$

Since $0 \leq(x-x \ln x) \leq 1$, for $0 \leq x \leq 1$, the second term in (17) is dominated by $\left\|g^{(2)}\right\|_{r^{\prime}}$ for all $0 \leq r^{\prime} \leq r$, so that, using Lemma 5 again,

$$
\left|\int_{0}^{1} d u \frac{g(E+u)-g(E-u)}{u}\right| \leq\left(\frac{8}{\pi}+2\right) \frac{1}{r^{2}}\|g\|_{r} .
$$

The second part is simply dominated by

$$
\left|\int_{1}^{\infty} d u \frac{g(E+u)-g(E-u)}{u}\right| \leq\|g\|_{r} .
$$

Thanks to Lemma 5 again, the last term on the right in (16) is bounded by

$$
|\imath \pi g(E)| \leq \frac{1}{r}\|g\|_{r}
$$

Overall, this gives

$$
\left|I_{0}(g ; E \pm \imath 0)\right| \leq\left(\left(\frac{8}{\pi}+2\right) \frac{1}{r^{2}}+\frac{1}{r}+1\right)\|g\|_{r}
$$




\section{Analyticity of the Density of States}

In order to illustrate our technique in the simplest setting, we prove the analyticity of the density of states (DOS) in the strong disorder regime. This is a new and different proof of a result in [7] obtained that was obtained using the supersymmetric replica trick for the Green's function. It is an improvement of the result of Constantinescu, Fröhlich, and Spencer [10]. The case $N=1$ requires only the Cauchy-type integral estimates of section 4. The DOS exists as a function in $L_{l o c}^{1}(\mathbb{R})$ for lattice models since the integrated density of states is globally Lipschitz continuous. The main result of this section is the following theorem.

Theorem 2 Let $H_{\omega}$ be the Anderson Hamiltonian defined in (1) with a distribution $g \in$ $\mathcal{H}_{r}$, for some $r>0$. Then, for any $0<\epsilon<r$, there is $\lambda_{r, \epsilon}>0$ such that for all $0 \leq \lambda<\lambda_{r, \epsilon}$, the DOS for $H_{\omega}$ is analytic in a strip of width $r-\epsilon>0$ about the real axis.

In order to prove Theorem 2, we recall that the DOS $\rho(E)$ is given by

$$
\begin{aligned}
\rho(E) & =\int_{\Omega} d \mathbb{P}\left\langle 0\left|\rho_{\omega}(E)\right| 0\right\rangle \\
& =\mathbb{E}\{\langle 0|\Im G(E+i 0)| 0\rangle\} \\
& =\lim _{\epsilon \rightarrow 0} \frac{1}{2 i}\left\{G_{\underline{1}}(E+i \epsilon)-G_{\underline{1}}(E-i \epsilon)\right\},
\end{aligned}
$$

using the notation of (8), and $\rho_{\omega}(E)$ is the spectral function for $H_{\omega}$ introduced in (2). We consider functions the $G_{1}(E \pm i \epsilon) \equiv G_{1}^{ \pm}(E)$ appearing in (18). We prove that each function is real analytic on $\mathbb{R}$ and that each has a holomorphic continuation to the lower, respectively, upper, half complex plane in a strip of size $r-\epsilon$, provided $|\lambda|$ is small enough. Using the random walk expansion described in section 2 , we find

$$
G_{\underline{1}}(z)=\sum_{\gamma: 0 \rightarrow 0}(-\lambda)^{|\gamma|} \prod_{u \in \mathbb{Z}^{d}} I_{n_{\gamma}(u)-1}(g ; z),
$$

where the Cauchy-type integral $I_{n_{\gamma}(u)}(g ; z)$ is given by

$$
I_{n}(g ; z) \equiv \int_{-\infty}^{\infty} d v \frac{g(v)}{(v-z)^{n+1}},
$$

as defined in (15). With reference to Section 6, we note that

$$
J_{n}^{ \pm}(g ; E) \equiv \lim _{\epsilon \rightarrow 0} \frac{1}{n !} I_{0}\left(g^{(n)} ; E \pm i \epsilon\right) .
$$


Lemma 9 The function $J_{n}^{ \pm}(g ; E)$, defined in (20), is analytic on $|\Im E|<r$ and its derivatives have the form

$$
\frac{1}{l !} \frac{\partial^{l}}{\partial E^{l}} J_{n}^{ \pm}(g ; E)=\frac{(n+l) !}{n ! l !} I_{0}^{ \pm}\left(\frac{g^{(n+l)}}{(n+l) !} ; E\right)
$$

where

$$
I_{0}^{ \pm}(h ; E) \equiv \lim _{\epsilon \rightarrow 0} I_{0}(h ; E \pm i \epsilon) .
$$

Proof. The formula (21) follows from Lemma 6] and Lemma 7 . The analyticity follows from the hypotheses on $g$ and the explicit formula in Lemma 7.

In order to prove real analyticity, we prove that the sum of terms on the left side of (21) converges uniformly. For this it is sufficient to estimate the sum

$$
\left\|J_{n}^{ \pm}(g ; \cdot)\right\|_{\delta} \equiv \sum_{l=0}^{\infty} \frac{\delta^{l}}{l !} \sup _{E \in \mathbb{R}}\left|\frac{\partial^{l}}{\partial E^{l}} J_{n}^{ \pm}(g ; E)\right|,
$$

for $0<\delta<r$. Since the diameter $\delta$ will be shown to be independent of the energy, it follows that the sum converges to an analytic function for $|\Im E|<\delta$.

Lemma 10 For $0<\delta<r$, the following estimate holds

$$
\left\|J_{n}^{ \pm}(g ; \cdot)\right\|_{\delta} \leq \frac{r e^{2} C}{4} \frac{(n+3)^{2}}{(r-\delta)^{n+3}}\|g\|_{r},
$$

with $C=(8 / \pi)+2+r+r^{2}$.

Proof. Lemma 7. Lemma 8 and then Lemma 5 lead to

$$
\left\|I_{0}^{ \pm}\left(\frac{g^{(l+n)}}{(n+l) !} ; \cdot\right)\right\|_{\infty} \leq \frac{1}{r_{1}^{n+l}} \frac{C\|g\|_{r}}{\left(r-r_{1}\right)^{2}},
$$

for any $0<r_{1}<r$. Summing over $l$ and using formula (35) leads to a convergent sum provided $\delta<r_{1}<r$ such that

$$
\left\|J_{n}^{ \pm}(g ; \cdot)\right\|_{\delta} \leq \frac{r_{1}}{\left(r_{1}-\delta\right)^{n+1}} \frac{C\|g\|_{r}}{\left(r-r_{1}\right)^{2}} .
$$

Maximizing the denominator on the right side of (23) over $r_{1}$, gives the result.

Proof of Theorem 2. Anticipating Section [7. Lemma 15 implies that the norm $\|\cdot\|_{\delta}$, defined in eq. (22), is multiplicative. Thus, from the path expansion (19), it follows that

$$
\left\|G_{\underline{1}}^{ \pm}(\cdot)\right\|_{\delta} \leq \sum_{\gamma: 0 \rightarrow 0}|\lambda|^{|\gamma|} \prod_{u \in \mathbb{Z}^{d}}\left\|J_{n_{\gamma}(u)-1}^{ \pm}\right\|_{\delta}
$$


The bound $(n / 2+1)^{2} \leq e^{n}$ implies $\prod_{u \in \mathcal{V}(\gamma)}\left(n_{\gamma}(u) / 2+1\right) \leq e^{|\gamma|}$. Definition (22) and Lemma 10] lead to

$$
\left\|G_{\underline{1}}^{ \pm}(\cdot)\right\|_{\delta} \leq \sum_{\gamma: 0 \rightarrow 0}|\lambda|^{|\gamma|}\left[\frac{r e^{2} C\|g\|_{r}}{(r-\delta)^{2}}\right]^{|\mathcal{V}(\gamma)|} \frac{e^{|\gamma|}}{(r-\delta)^{|\gamma|}} .
$$

Using the inequality $|\mathcal{V}(\gamma)| \leq|\gamma|+1$ this leads to

$$
\left\|G_{\underline{1}}^{ \pm}(\cdot)\right\|_{\delta} \leq \frac{r e^{2} C\|g\|_{r}}{(r-\delta)^{2}} \sum_{\gamma: 0 \rightarrow 0}\left[\frac{|\lambda| r e^{3} C\|g\|_{r}}{(r-\delta)^{3}}\right]^{|\gamma|} .
$$

Finally, from Lemma 4, the number of paths of length $n$ is bounded by $(2 d)^{n}$ so the sum over paths can be changed into a sum over $n$ to get

$$
\left\|G_{\underline{1}}^{ \pm}(\cdot)\right\|_{\delta} \leq \frac{r e^{2} C\|g\|_{r}}{(r-\delta)^{2}} \sum_{n \geq 0}\left[\frac{2 d|\lambda| r e^{3} C\|g\|_{r}}{(r-\delta)^{3}}\right]^{n} .
$$

So for any small $\epsilon>0$, if $\lambda_{r, \epsilon} \equiv \epsilon^{3} /\left(2 d e^{3} r C\|g\|_{r}\right)$, then for any $|\lambda|<\lambda_{r, \epsilon}$, the DOS is analytic in the strip $|\Im E|<r-\epsilon$.

\section{Estimates on $N$-point Cauchy-type Integrals}

In this section, the general $N$-point Cauchy-type integrals appearing in (13)-(14) is considered. Results similar to those of section 3 for the case of $N=1$ will be obtained. Let $\underline{n}=\left(n_{1}, \cdots, n_{N}\right) \in \mathbb{N}^{N}$, and let $\underline{z}=\left(z_{1}, \cdots, z_{N}\right) \in(\mathbb{C} \backslash \mathbb{R})^{N}$. The following integral will be considered

$$
J_{\underline{n}}(g ; \underline{z})=\int_{-\infty}^{+\infty} d v \frac{g(v)}{\left(v-z_{1}\right)^{n_{1}+1} \cdots\left(v-z_{N}\right)^{n_{N}+1}},
$$

with the following usual convention for multi-indices

$$
\underline{n} !=\prod_{k=1}^{N} n_{k} !, \quad|\underline{n}|=\sum_{k=1}^{N} n_{k}, \quad \partial_{\underline{z}}^{\underline{n}}=\prod_{k=1}^{N} \frac{\partial^{n_{k}}}{\partial z_{k}^{n_{k}}} .
$$

Lemma 11 The following formula hold

$$
J_{\underline{n}}(g ; \underline{z})=\frac{1}{\underline{n} !} \partial^{\underline{n}} J_{\underline{0}}(g ; \underline{z})
$$

and

$$
J_{\underline{0}}(g ; \underline{z})=\sum_{i=1}^{N} \prod_{j: \neq i} \frac{1}{z_{i}-z_{j}} I_{0}\left(g ; z_{i}\right)
$$


Proof: The first formula (25) is obtained by using repetitively the identity

$$
\frac{1}{(v-z)^{n+1}}=\frac{1}{n !} \frac{d^{n}}{d z^{n}} \frac{1}{v-z} .
$$

The formula (26) is obtained from the following identity, valid if $z_{k} \neq z_{l}$ for $k \neq l$

$$
\frac{1}{\left(v-z_{1}\right) \cdots\left(v-z_{N}\right)}=\sum_{i=1}^{N} \prod_{j: \neq i} \frac{1}{z_{i}-z_{j}} \frac{1}{v-z_{i}}
$$

Lemma 12 The following formula holds

$$
J_{\underline{n}}(g ; \underline{z})=\sum_{i=1}^{N} \sum_{\underline{m} ;|\underline{m}|=n_{i}} \prod_{j: \neq i} \frac{(-1)^{m_{j}}\left(m_{j}+n_{j}\right) !}{m_{j} ! n_{j} !} \frac{1}{\left(z_{i}-z_{j}\right)^{m_{j}+n_{j}+1}} \frac{1}{m_{i} !} I_{0}\left(g^{\left(m_{i}\right)} ; z_{i}\right) .
$$

Proof: Applying (25) to (26) gives

$$
J_{\underline{n}}(g ; \underline{z})=\sum_{i=1}^{N} \frac{1}{n_{i} !} \frac{\partial^{n_{i}}}{\partial z_{i}^{n_{i}}}\left\{\prod_{j: \neq i} \frac{1}{\left(z_{i}-z_{j}\right)^{n_{j}+1}} I_{0}\left(g ; z_{i}\right)\right\} .
$$

The following multiple variable generalization of the Leibnitz formula will be used

$$
\frac{1}{r !} \frac{d^{r}}{d z^{r}} f_{1}(z) \cdots f_{N}(z)=\sum_{\underline{m} ;|\underline{m}|=r} \prod_{k=1}^{N} \frac{1}{m_{k} !} \frac{d^{m_{k}} f_{k}}{d z^{m_{k}}}
$$

Applying it to the previous formula, together with (27), leads to the result.

The analyticity properties of these functions and their boundary values is now investigated in a manner similar to Lemma 7 . For $\underline{\sigma} \in\{+,-\}^{N}$, let $J \underline{\underline{\sigma}}(g ; \underline{E})$ be defined by

$$
J_{\underline{\underline{n}}}(g ; \underline{E})=\lim _{\epsilon_{k} \downarrow 0} J_{\underline{n}}\left(g ; E_{1}+\imath \sigma_{1} \epsilon_{1}, \cdots, E_{N}+\imath \sigma_{N} \epsilon_{N}\right) .
$$

Then, as a consequence of (29)

Lemma 13 If $\underline{\sigma} \in\{+,-\}^{N}$, the function $\underline{E} \in \mathbb{R}^{N} \mapsto J \underline{\underline{\sigma}}(g ; \underline{E}) \in \mathbb{C}$ is analytic away from coincident points. Moreover, away from coincident points,

$$
\begin{aligned}
\frac{1}{\underline{l} !} \partial^{\underline{l}} J_{\underline{\underline{n}}}(g ; \underline{E})= & \sum_{i=1}^{N} \sum_{\underline{m} ;|\underline{m}|=l_{i}+n_{i}}\left\{\prod_{j: \neq i} \frac{(-1)^{m_{j}}\left(m_{j}+l_{j}+n_{j}\right) !}{m_{j} ! l_{j} ! n_{j} !} \frac{1}{\left(E_{i}-E_{j}\right)^{m_{j}+l_{j}+n_{j}+1}}\right\} \\
& \times \frac{\left(n_{i}+l_{i}\right) !}{l_{i} ! n_{i} ! m_{i} !} I_{0}^{\sigma_{i}}\left(g^{\left(m_{i}\right)} ; E_{i}\right),
\end{aligned}
$$


where

$$
I_{0}^{\sigma_{i}}\left(h ; E_{i}\right)=\lim _{\epsilon_{i} \downarrow 0} I_{0}\left(h ; E_{i}+i \sigma_{i} \epsilon_{i}\right) .
$$

Proof: Using equations (25) and (29) leads directly to this formula. The real analyticity comes from the use of the contour of integration used in the proof of Lemma 7 provided each semicircle has radius satisfying $0<\epsilon_{i}<r$.

Let $\Delta_{N}$ be the usual $(N-1)$-simplex, namely, the set of $\underline{s}=\left(s_{1}, \cdots, s_{N}\right) \in[0,1]^{N}$ such that $|\underline{s}|=s_{1}+\cdots+s_{N}=1$. Let $d^{N-1} \underline{s}$ denote the measure $d s_{1} \cdots d s_{N-1}$ defined on $\Delta_{N}$, and set $\underline{s}^{\underline{n}}=\prod_{k=1}^{N} s_{k}^{n_{k}}$. The following result generalizes Lemma 7 and eq. [16]

Proposition 2 The function $\underline{E} \in \mathbb{R}^{N} \mapsto J \underline{\underline{\sigma}}(g ; \underline{E}) \in \mathbb{C}$ is analytic away from coincident points and

$$
J_{\underline{n}}^{\underline{\sigma}}(g ; \underline{E})=J_{\underline{n}}^{r e g}(g ; \underline{E})+\imath \pi \sum_{k=1}^{N} \sum_{\underline{m},|\underline{m}|=n_{k}} \frac{\sigma_{k} g^{\left(m_{k}\right)}\left(E_{k}\right)}{m_{k} !} \prod_{j: \neq k} \frac{1}{\left(E_{k}-E_{j}\right)^{n_{j}+m_{j}+1}},
$$

where $\underline{E} \in \mathbb{R}^{N} \mapsto J=J_{\underline{n}}^{r e g}(g ; \underline{E}) \in \mathbb{C}$ is analytic everywhere and given by

$$
J=\int_{\Delta_{N}} d^{N-1} \underline{s}\left(\frac{\underline{s}^{\underline{n}}}{\underline{n} !}\right) \int_{0}^{\infty} d u \frac{g^{(N+|\underline{n}|-1)}\left(\sum_{k} s_{k} E_{k}+u\right)-g^{(N+|\underline{n}|-1)}\left(\sum_{k} s_{k} E_{k}-u\right)}{u} .
$$

In addition, if $\sigma_{1}=\cdots=\sigma_{N}= \pm 1$, the function $\underline{E} \in \mathbb{R}^{N} \mapsto J \underline{\underline{\sigma}}(g ; \underline{E}) \in \mathbb{C}$ is analytic everywhere and given by

$$
\sigma_{1}=\cdots=\sigma_{N}= \pm 1 \quad \Rightarrow \quad J \underline{\underline{\sigma}}(g ; \underline{E})=J_{\underline{\underline{n}}}^{r e g}(g ; \underline{E}) \pm \imath \pi R_{\underline{n}}(g ; \underline{E}),
$$

with

$$
R_{\underline{n}}(g ; \underline{E})=\int_{\Delta_{N}} d^{N-1} \underline{s}\left(\frac{\underline{s}^{\underline{n}}}{\underline{n} !}\right) g^{(N+|\underline{n}|-1)}\left(\sum_{k} s_{k} E_{k}\right) .
$$

Remark 1 The previous proposition shows that $J_{\underline{n}}^{\frac{\sigma}{n}}$ admits a polar singularity at $E_{k}=E_{j}$ if and only if $\sigma_{k} \neq \sigma_{j}$.

The first step in the proof is a simple lemma relating integration over an $N-1$-simplex to the product of the singular terms in the integrand in (24). Given a set of points $\left\{z_{1}, \cdots, z_{N}\right\}$, their convex hull will be denoted by $\operatorname{Conv}\left\{z_{1}, \cdots, z_{N}\right\}$. 
Lemma 14 Let $\underline{n} \in \mathbb{N}^{N}$ and $\underline{z} \in(\mathbb{C} \backslash \mathbb{R})^{N}$. If $v \notin \operatorname{Conv}\left\{z_{1}, \cdots, z_{N}\right\}$, the following formula holds

$$
\prod_{k=1}^{N} \frac{1}{\left(v-z_{k}\right)^{n_{k}+1}}=\frac{(N+|\underline{n}|-1) !}{\underline{n} !} \int_{\Delta_{N}} d^{N-1} \underline{s} \frac{\underline{s}^{\underline{n}}}{\left(v-\sum_{k=1}^{N} s_{k} z_{k}\right)^{N+|\underline{n}|}} .
$$

Proof: Both sides of this formula are defined and holomorphic in $v$ in the domain $\left\{v \in \mathbb{C} ; v \notin \operatorname{Conv}\left\{z_{1}, \cdots, z_{N}\right\}\right\}$. Thus, using the unique analytic continuation theorem, it is sufficient to prove it for $\Re\left(v-z_{k}\right)>0$. Setting $a_{k}=v-z_{k}$, the identity

$$
\frac{1}{a_{k}^{n_{k}+1}}=\int_{0}^{\infty} d t_{k} \frac{t_{k}^{n_{k}}}{n_{k} !} e^{-t_{k} a_{k}}
$$

valid for $\Re a_{k}>0$, implies

$$
\prod_{k=1}^{N} \frac{1}{\left(a_{k}\right)^{n_{k}+1}}=\int_{\mathbb{R}_{+}^{N}} d^{N} \underline{t} \frac{\underline{t}^{\underline{n}}}{\underline{n} !} e^{-\sum_{k} t_{k} a_{k}} .
$$

The following change of variables $\left(t_{1}, \cdots, t_{N}\right) \mapsto\left(\lambda, s_{1}, \cdots, s_{N-1}\right)$ will be useful

$$
t_{k}=\lambda s_{k}, \quad(1 \leq k \leq N) \quad s_{N}=1-\sum_{k=1}^{N-1} s_{k}, \lambda \geq 0 .
$$

In particular, we have $s_{k} \geq 0 \forall k$, and $s_{1}+\cdots+s_{N-1} \leq 1$. Furthermore, the volume elements transform as

$$
d t_{1} \wedge \cdots \wedge d t_{N}=(-1)^{N-1} \lambda^{N-1} d \lambda \wedge d s_{1} \wedge \cdots \wedge d s_{N-1}
$$

so that

$$
\begin{aligned}
\prod_{k=1}^{N} \frac{1}{\left(a_{k}\right)^{n_{k}+1}} & =\int_{\Delta_{N}} d s_{1} \cdots d s_{N-1} \frac{\underline{s}^{\underline{n}}}{\underline{n} !} \int_{0}^{\infty} \lambda^{N+|\underline{n}|-1} d \lambda e^{-\lambda \sum_{k} s_{k} a_{k}} \\
& =\int_{\Delta_{N}} d s_{1} \cdots d s_{N-1} \frac{\underline{s}^{\underline{n}}}{\underline{n} !} \frac{(N+|\underline{n}|-1) !}{\left(\sum_{k} s_{k} a_{k}\right)^{N+|\underline{n}|}}
\end{aligned}
$$

Replacing $a_{k}$ by $v-z_{k}$ gives the result.

\section{Proof of Proposition 2:}

1. The analyticity claim in the first part of the proposition follows once we have proved the representations (31) and (32). If $z_{1}, \cdots, z_{N}$ are all on the same side of the real axis, then the convex hull is also contained in the same half plane. Thanks to Lemma 14, it follows that 


$$
\begin{aligned}
J_{n_{1}, \cdots, n_{N}}\left(g ; z_{1}, \cdots, z_{N}\right) & =\int_{\Delta_{N}} d^{N-1} \underline{s} \frac{\underline{s}^{\underline{n}}}{\underline{n} !} \int_{-\infty}^{+\infty} d v \frac{g(v)(N+|\underline{n}|-1) !}{\left(v-\sum_{k} s_{k} z_{k}\right)^{N+|\underline{n}|}} \\
& =\int_{\Delta_{N}} d^{N-1} \underline{s} \frac{\underline{s}^{\underline{n}}}{\underline{n} !} I_{0}\left(g^{(N+|\underline{n}|-1)} ; \sum_{k} s_{k} z_{k}\right)
\end{aligned}
$$

From Lemma 7 equations (32), (33), and (34) follow immediately.

2. To derive (31) from (34) in the case $\sigma_{1}=\cdots=\sigma_{N}= \pm 1$, we apply Lemma 14 for $n_{1}=\cdots=n_{N}=1$, together with (28), to obtain

$$
\int_{\Delta_{N}} d^{N-1} \underline{s} \frac{(N-1) !}{\left(z-\sum_{k} s_{k} E_{k}\right)^{N}}=\frac{1}{\left(z-E_{1}\right) \cdots\left(z-E_{N}\right)}=\sum_{k=1}^{N} \frac{1}{z-E_{k}} \prod_{j: \neq k} \frac{1}{E_{k}-E_{j}} .
$$

Multiplying both sides by $g(z)$ and integrating over a Jordan path surrounding each $E_{k}$ once and contained in the holomorphy domain of $g$, leads to

$$
\int_{\Delta_{N}} d^{N-1} \underline{s} g^{(N-1)}\left(\sum_{k} s_{k} E_{k}\right)=\sum_{k=1}^{N} g\left(E_{k}\right) \prod_{j: \neq k} \frac{1}{E_{k}-E_{j}} .
$$

Applying the differential operator $(1 / \underline{n} !) \partial_{\underline{E}}^{\underline{n}}$ on both sides gives

$$
\int_{\Delta_{N}} d^{N-1} \underline{s} \frac{\underline{s}^{\underline{n}}}{\underline{n} !} g^{(N+|\underline{n}|-1)}\left(\sum_{k} s_{k} E_{k}\right)=\sum_{k=1}^{N} \frac{1}{n_{k}} \partial_{E_{k}}^{n_{k}}\left(g\left(E_{k}\right) \prod_{j: \neq k} \frac{1}{\left(E_{k}-E_{j}\right)^{n_{j}+1}}\right) .
$$

An application of Leibnitz rule (see (30) ) gives equation (31) for $\sigma_{1}=\cdots=\sigma_{N}=1$.

3. If $\underline{\sigma}=\left(\sigma_{1}, \cdots, \sigma_{N}\right) \in\{+1,-1\}^{N}$ is arbitrary, let $A(\underline{\sigma}) \subset[1, N]$ be the set of indices $k$ such that $\sigma_{k}=-1$. Whenever $\underline{z}=\left(z_{1}, \cdots, z_{N}\right) \in \mathbb{C}^{N}$ is such that $\sigma_{k} \Im z_{k}>0$ for all $k$ 's, the integration contour defining $J_{\underline{n}}(g ; \underline{z})$ cannot be deformed to have all $z_{k}$ 's on the same side. Actually the $z_{k}$ 's such that $k \in A(\underline{\sigma})$ are below the integration path while the other $z_{k}$ 's are above. Deforming the contour to get all the $z_{k}$ 's above can be done to the price of adding the corresponding residues, leading to

$$
\begin{aligned}
J_{\underline{n}}(\underline{E}) & =J_{\underline{n}}^{+}(\underline{E})-2 \imath \pi \sum_{k \in A(\underline{\sigma})} \operatorname{Res}_{z=E_{k}} \frac{g(z)}{\left(z-E_{1}\right)^{n_{1}+1} \cdots\left(z-E_{N}\right)^{n_{N}+1}} \\
& =J_{\underline{n}}^{+}(\underline{E})+\imath \pi \sum_{k=0}^{N}\left(\sigma_{k}-1\right) \operatorname{Res}_{z=E_{k}} \frac{g(z)}{\left(z-E_{1}\right)^{n_{1}+1} \cdots\left(z-E_{N}\right)^{n_{N}+1}} .
\end{aligned}
$$

The residues are given by 


$$
\operatorname{Res}_{z=E_{k}} \frac{g(z)}{\left(z-E_{1}\right)^{n_{1}+1} \cdots\left(z-E_{N}\right)^{n_{N}+1}}=\frac{1}{n_{k} !} \partial_{E_{k}}^{n_{k}}\left(g\left(E_{k}\right) \prod_{j: \neq k} \frac{1}{\left(E_{k}-E_{j}\right)^{n_{j}+1}}\right)
$$

Use of the Leibnitz rule again together with the previous formula for $J_{\underline{n}}^{+}(g ; \underline{E})$ gives (31) in the general case.

\section{Real Analyticity}

We now use the estimates on the Cauchy integrals obtained in Section [6 in order to prove that the $N$-point Cauchy-type integrals, for $N \geq 2$, are real analytic as functions of $\underline{E} \in \mathbb{N}^{N}$, on the domain for which $E_{j} \neq E_{i}$, for $i \neq j$. To this end, we prove that the functions $J \underline{\underline{a}}(g ; \underline{E})$, defined in section 6 , have uniformly convergent power series on appropriate domains. It is convenient to do this using certain Banach spaces of real analytic functions. Let $\delta>0$ and let $\mathcal{U}$ be an open subset of $\mathbb{R}^{N}$. For a continuous complex valued function $f$ on $\mathcal{U}$ let $\|f\|_{\mathcal{U}}$ be the sup-norm $\|f\|_{\mathcal{U}}=\sup _{\underline{E} \in \mathcal{U}}|f(\underline{E})|$. Then let $\mathcal{E}_{\delta}(\mathcal{U})$ be the space of smooth functions $F: \underline{E} \in \mathcal{U} \mapsto \mathbb{C}$ vanishing at infinity, together with all derivatives, such that

$$
\|F\|_{\delta, \mathcal{U}}=\sum_{\underline{\underline{l}} \in \mathbb{N}^{N}} \frac{\delta^{|\underline{l}|}}{\underline{l} !}\left\|\partial^{\underline{l}} F\right\|_{\mathcal{U}}<\infty
$$

Endowed with the norm $\|\cdot\|_{\delta, \mathcal{U}}$ the space $\mathcal{E}_{\delta}(\mathcal{U})$ is a Banach space. Moreover, it is a Banach $*$-algebra if endowed with the pointwise multiplication and complex conjugacy since

Lemma 15 Let $F$ and $G$ be two elements of $\mathcal{E}_{\delta}(\mathcal{U})$. Then both $F^{*}$ and $F \cdot G$ belong to $\mathcal{E}_{\delta}(\mathcal{U})$ and

$$
\|F \cdot G\|_{\delta, \mathcal{U}} \leq\|F\|_{\delta, \mathcal{U}}\|G\|_{\delta, \mathcal{U}}, \quad \quad\left\|F^{*}\right\|_{\delta, \mathcal{U}}=\|F\|_{\delta, \mathcal{U}}
$$

Proof: The only non trivial property is the first inequality. Using the Leibnitz rule (see (301) ) leads to

$$
\frac{1}{\underline{l} !} \partial^{\underline{l}}(F \cdot G)=\sum_{\underline{m}, \underline{n} ; \underline{m}+\underline{n}=\underline{l} \underline{\underline{m}} !} \frac{1}{\underline{m}} \partial^{\underline{m}} F \frac{1}{\underline{n} !} \partial^{\underline{n}} G .
$$

From this the inequality follows immediately since $\delta^{|\underline{m}+\underline{n}|}=\delta^{|\underline{m}|} \delta^{|\underline{n}|}$.

When we take $\mathcal{U}=\mathbb{R}^{N}$, we write $\|\cdot\|_{\delta}$ in place of $\|\cdot\|_{\delta, \mathbb{R}^{N}}$. 
Proposition 3 Let $g$ be an element of $\mathcal{H}_{r}$, for some $r>0$, let $C=8 / \pi+2+r+r^{2}$, and consider $N \geq 2$. For any choice of $\delta>0$ and $\Delta>0$ such that $0<\delta<\Delta / 2$ and $0<\Delta-\delta<r / 2$, the boundary values of $J_{\underline{\underline{\sigma}}}^{\underline{\sigma}}(g)$ satisfy the following estimate

$$
\left\|J_{\underline{\underline{a}}}(g)\right\|_{\delta, \mathcal{D}_{\Delta}} \leq \frac{4 C N\|g\|_{r}}{r}\left(\frac{e}{\Delta-\delta}\right)^{|\underline{n}|+N} .
$$

in the domain $\mathcal{D}_{\Delta}=\left\{\underline{E} \in \mathbb{R}^{N} ;\left|E_{i}-E_{j}\right|>\Delta\right\}$.

The proof will proceed in several steps. First the following identity will be used often

$$
(1-u)^{-n-1}=\sum_{l=0}^{\infty} \frac{(l+n) !}{l ! n !} u^{l} .
$$

The following combinatorial estimate is necessary.

Lemma 16 If $\underline{n} \in \mathbb{N}^{L}$ and if $r \in \mathbb{N}$, then

$$
\sum_{|\underline{m}|=r} \frac{(\underline{m}+\underline{n}) !}{\underline{m} ! \underline{n} !}=\frac{(r+|\underline{n}|+L-1) !}{r !(|\underline{n}|+L-1) !}
$$

Proof: If $C_{r}^{L}(\underline{n})$ denotes the left side of (36), its definition and the identity (35) give

$$
\sum_{r=0}^{\infty} C_{r}^{L}(\underline{n}) X^{r}=\prod_{k=1}^{L} \sum_{m_{k}=0}^{\infty} \frac{\left(m_{k}+n_{k}\right) !}{m_{k} ! n_{k} !} X^{m_{k}}=\frac{1}{(1-X)^{|\underline{n}|+L}} .
$$

Expanding the right side in formal power series in $X$ by using (35) again gives directly the result.

Proof of Proposition [3; Using Lemma 13 and assuming $\left|E_{i}-E_{j}\right|>\Delta$ gives

$$
\begin{aligned}
\left\|J_{\underline{\underline{n}}}(g)\right\|_{\delta, \mathcal{D}_{\Delta}} \leq & \sum_{i=1}^{N} \sum_{\underline{\underline{l} \in \mathbb{N}^{N}}} \sum_{\underline{m} ;|\underline{m}|=l_{i}+n_{i}}\left\{\prod_{j: \neq i} \frac{\left(m_{j}+l_{j}+n_{j}\right) !}{m_{j} ! l_{j} ! n_{j} !}\left(\frac{\delta}{\Delta}\right)^{l_{j}} \frac{1}{\Delta^{m_{j}+n_{j}+1}}\right\} \\
& \times \frac{\left(l_{i}+n_{i}\right) !}{l_{i} ! n_{i} !} \delta^{l_{i}}\left\|I_{0}\left(\frac{g^{\left(m_{i}\right)}}{m_{i} !}\right)\right\|_{\infty} .
\end{aligned}
$$

Using the distributive property of the product with respect to addition for the variable $l_{j}$ gives

$$
\begin{aligned}
\left\|J_{\underline{\underline{\underline{\sigma}}}}(g)\right\|_{\delta, \mathcal{D}_{\Delta} \leq} \leq & \sum_{i=1}^{N} \sum_{l_{i}=0}^{\infty} \sum_{\underline{m} ; \mid \underline{\underline{m} \mid=l_{i}+n_{i}}}\left\{\prod_{j: \neq i}\left(\sum_{l_{j}=0}^{\infty} \frac{\left(m_{j}+l_{j}+n_{j}\right) !}{m_{j} ! l_{j} ! n_{j} !}\left(\frac{\delta}{\Delta}\right)^{l_{j}}\right) \frac{1}{\Delta^{m_{j}+n_{j}+1}}\right\} \\
& \times \frac{\left(l_{i}+n_{i}\right) !}{l_{i} ! n_{i} !} \delta^{l_{i}}\left\|I_{0}\left(\frac{g^{\left(m_{i}\right)}}{m_{i} !}\right)\right\|_{\infty} .
\end{aligned}
$$


We use Lemmas 5 and 8 to estimate the $L^{\infty}$-norm in (37) for any $0<r_{i}<r$ by

$$
\left\|I_{0}\left(\frac{g^{\left(m_{i}\right)}}{m_{i} !}\right)\right\|_{\infty} \leq \frac{C}{\left(r-r_{i}\right)^{2}} \frac{\left\|g^{\left(m_{i}\right)}\right\|_{r-r_{i}}}{m_{i} !}, \quad C=\frac{8}{\pi}+2+r+r^{2} .
$$

Using (35) to sum over $l_{j}$, together with estimate (38), leads to

$$
\begin{aligned}
\left\|J_{\underline{n}}(g)\right\|_{\delta, \mathcal{D}_{\Delta}} \leq & \sum_{i=1}^{N} \sum_{l_{i}=0}^{\infty} \sum_{\underline{m} ;|\underline{m}|=l_{i}+n_{i}}\left\{\prod_{j: \neq i} \frac{\left(m_{j}+n_{j}\right) !}{m_{j} ! n_{j} !} \frac{1}{(\Delta-\delta)^{m_{j}+n_{j}+1}}\right\} \\
& \times \frac{\left(l_{i}+n_{i}\right) !}{l_{i} ! n_{i} !} \frac{\delta^{l_{i}}}{r_{i}^{m_{i}}} \frac{C}{\left(r-r_{i}\right)^{2}}\|g\|_{r},
\end{aligned}
$$

for $0<r_{i}<r$. We define $p$ by $|\underline{m}|=m_{i}+p$ and $|\underline{n}|_{i}=|\underline{n}|-n_{i}$. With this definition and Lemma 16, we perform the restricted sum over $\underline{m} \in \mathbb{N}$ and obtain

$$
\begin{aligned}
\left\|J_{\underline{\underline{n}}}(g)\right\|_{\delta, \mathcal{D}_{\Delta}} \leq & \sum_{i=1}^{N} \frac{1}{(\Delta-\delta)^{|\underline{n}|_{i}+N-1}} \sum_{l_{i}=0}^{\infty}\left(\sum_{p=0}^{l_{i}+n_{i}} \frac{\left(p+|\underline{n}|_{i}+N-2\right) !}{p !\left(|\underline{n}|_{i}+N-2\right) !}\left(\frac{r_{i}}{\Delta-\delta}\right)^{p}\right) \\
& \times \frac{\left(l_{i}+n_{i}\right) !}{l_{i} ! n_{i} !} \frac{\delta^{l_{i}}}{r_{i}^{n_{i}+l_{i}}} \frac{C}{\left(r-r_{i}\right)^{2}}\|g\|_{r} .
\end{aligned}
$$

Using (35) again to sum over $p$, if $r_{i}<\Delta-\delta$, leads to

$$
\|J \underline{\underline{a}}(g)\|_{\delta, \mathcal{D}_{\Delta}} \leq \sum_{i=1}^{N} \frac{C\|g\|_{r}}{\left(r-r_{i}\right)^{2} r_{i}^{n_{i}}\left(\Delta-\delta-r_{i}\right)^{|\underline{n}|_{i}+N-1}} \sum_{l_{i}=0}^{\infty} \frac{\left(l_{i}+n_{i}\right) !}{l_{i} ! n_{i} !}\left(\frac{\delta}{r_{i}}\right)^{l_{i}}
$$

Finally, using again (35) to sum over $l_{i}$, if $\delta<r_{i}<r$, leads to

$$
\|J \underline{\underline{\sigma}}(g)\|_{\delta, \mathcal{D}_{\Delta}} \leq \sum_{i=1}^{N} \frac{C r\|g\|_{r}}{\left(r-r_{i}\right)^{2}\left(r_{i}-\delta\right)^{n_{i}+1}\left(\Delta-\delta-r_{i}\right)^{|\underline{n}|_{i}+N-1}} .
$$

These estimates are satisfied on the domain $\mathcal{D}_{\Delta}$ provided $0<\delta<r_{i}<r$, and $(\Delta-\delta)>0$. In particular, if we take $\Delta>2 \delta$. Recalling that we treat here $N \geq 2$, if $\Delta-\delta<r / 2$, it follows that $r_{i}<r / 2$, so that $r /\left(r-r_{i}\right)^{2}<4 / r$. Then, minimizing over $r_{i}$ in each term on the right side gives

$$
r_{i}=\frac{\left(n_{i}+1\right)(\Delta-\delta)+\left(|\underline{n}|_{i}+N-1\right) \delta}{|\underline{n}|+N},
$$

leading to 


$$
r_{i}-\delta=(\Delta-\delta) \frac{n_{i}+1}{|\underline{n}|+N}, \quad \Delta-\delta-r_{i}=(\Delta-\delta) \frac{|\underline{n}|_{i}+N-1}{|\underline{n}|+N} .
$$

If $l, m$ are positive integers, $((l+m) / l)^{l}=(1+m / l)^{l} \leq e^{m}$, so that

$$
\left(\frac{l+m}{l}\right)^{l}\left(\frac{l+m}{m}\right)^{m} \leq e^{l+m}
$$

we obtain

$$
\|J \underline{\underline{a}}(g)\|_{\delta, \mathcal{D}_{\Delta}} \leq \frac{4 N C\|g\|_{r}}{r}\left(\frac{e}{\Delta-\delta}\right)^{|\underline{n}|+N}, \quad \text { if } \quad 0<\delta<\Delta-\delta<\frac{r}{2} .
$$

This proves Proposition 3.

\section{Proof of Theorem 1}

It is now possible to finish the proof of Theorem 1 proving the main theorem on the real analyticity of the $N$-point correlation functions $G_{\underline{A}}(\underline{E})$, away from a small neighborhood of the coincident points $E_{i}=E_{j}$. We recall the $\underline{A}$-compatible path expansion in (14). Our strategy is to write $G_{\underline{A}}(\underline{E})$ as a sum over $n \in \mathbb{N}$, the length of the compatible paths. We then prove that $G_{\underline{A}}(\underline{E})$ is in the Banach space $\mathcal{E}_{\delta}\left(\mathcal{D}_{\Delta}\right)$ using the estimates in Proposition 3. We first prove the theorem for the case when the covariant operators $A_{i}$ are $r$-monomials which implies the result when the covariant operators $A_{i}$ are $r$-polynomials. Starting from (14), it is sufficient to prove that the formal path expansion converges in $\mathcal{E}_{\delta}\left(\mathcal{D}_{\Delta}\right)$, with the domain $\mathcal{D}_{\Delta} \subset \mathbb{R}^{N}$, for convenient values of $\delta$ and $\Delta$. Using Proposition 3 , the $N$-point correlation $G \underline{\underline{A}}$ is estimated as follows

$$
\left\|G_{\underline{\underline{A}}}^{\underline{\sigma}}\right\|_{\delta, \mathcal{D}_{\Delta}} \leq \sum_{\Gamma \in \mathcal{P}(\underline{A})}|\lambda|^{|\Gamma|} \prod_{u \in \mathcal{V}(\Gamma)} N C_{1}\left\|g_{\Gamma, u}\right\|_{r}\left(\frac{e}{\Delta-\delta}\right)^{\left|\underline{n}_{\Gamma}(u)-\underline{f}\right|+N}, \quad C_{1}=\frac{4 C}{r} .
$$

Clearly, we have that $\left|\underline{n}_{\Gamma}(u)-\underline{f}\right|+N=\left|\underline{n}_{\Gamma}(u)\right|$. This is bounded above by the length of the path $|\Gamma|$. On the other hand, if $A=\max _{i, u}\left\|a_{i, u}\right\|$, then $A$ appears at most $N$-times in the right hand side in the definition of $g_{\Gamma, u}$, reflecting the fact that $G_{\underline{A}}$ is homogeneous of degree $N$ in $\underline{A}$. As a consequence, we get the bound

$$
\prod_{u \in \mathcal{V}(\Gamma)} N C_{1}\left\|g_{\Gamma, u}\right\|_{r} \leq A^{N} \prod_{u \in \mathcal{V}(\Gamma)} N C_{1}\|g\|_{r}
$$

On the other hand, since $g$ defines a probability on $\mathbb{R},\|g\|_{r} \geq 1$. Moreover, $C_{1}=$ $4(8 / \pi+2) / r+4+4 r \geq 4$, so that $N C_{1}\|g\|_{r}>1$. Hence, using $\# \Gamma \leq|\Gamma|$, and the Lemma 4, this inequality becomes 


$$
\begin{aligned}
\| G_{\underline{A}}^{\frac{\sigma}{A} \|_{\delta, \mathcal{D}_{\Delta}}} & \leq A^{N} \sum_{\Gamma \in \mathcal{P}(\underline{A})}\left(\frac{e|\lambda|}{\Delta-\delta}\right)^{|\Gamma|} \prod_{u \in \mathcal{V}(\Gamma)} N C_{1}\|g\|_{r} \leq A^{N} \sum_{\Gamma \in \mathcal{P}(\underline{A})}\left(\frac{e|\lambda| N C_{1}\|g\|_{r}}{\Delta-\delta}\right)^{|\Gamma|} \\
& \leq A^{N} \sum_{n=0}^{\infty}\left(\frac{2 d N C_{1} e|\lambda|\|g\|_{r}}{\Delta-\delta}\right)^{n} \leq \frac{A^{N}}{1-2 d N C_{1} e|\lambda|\|g\|_{r}(\Delta-\delta)^{-1}}<\infty,
\end{aligned}
$$

provided

$$
|\lambda|<\frac{\Delta-\delta}{2 d N C_{1} e\|g\|_{r}} .
$$

Choosing $\delta<\Delta / 2$, we define $a_{0} \equiv 4 d N C_{1} e\|g\|_{r}$. Hence, the function $G_{A}^{\underline{\sigma}} \in \mathcal{E}_{\delta}\left(\mathcal{D}_{\Delta}\right)$, provided $a_{0}|\lambda|<\Delta$, proving Theorem 1 for the case when $\underline{A}$ is a covariant family of $r$-monomials. We now consider a general covariant family $\underline{A} \in L^{\infty}\left(\mathcal{T}_{\mathbb{P}}\right)^{\times N}$ and the associated correlation function $G_{\underline{A}}(\underline{z})$, with $\Im z_{j}>0$. By Proposition $1, G_{\underline{A}}(\underline{z})$ can be approximated uniformly on any compact subset of $(\mathbb{C} \backslash \mathbb{R})^{N}$ by a sequence of correlation functions $G_{\underline{A}_{n}}(\underline{z})$, where the family $\underline{A}_{n}$ are $r$-polynomials. Furthermore, the coefficients can be chosen so that they are uniformly bounded in $L^{\infty}(\Omega, \mathbb{P})$. This family of correlation functions has an analytic continuation to $\mathcal{D}_{\Delta}$. Estimate (39) show that this family of approximating correlations functions is uniformly bounded since the bound depends on the coefficients. Since the family converges uniformly on any compact subset of the original domain $(\mathbb{C} \backslash \mathbb{R})^{N}$, the family converges uniformly on any compact subset of the extended domain in $\mathbb{C}^{N}$. The limit functions provide a continuation of $G_{\underline{A}}(\underline{z})$ into this extended domain by the identity principle for analytic functions. This proves Theorem 1.

\section{References}

[1] J. Bellissard, " $K$-Theory of $C^{*}$-algebras in Solid State Physics" in Statistical Mechanics and Field Theory, Mathematical Aspects, T.C. Dorlas, M.N. Hugenholtz \& M. Winnink, Lecture Notes in Physics, 257, (1986), 99-156.

[2] J. Bellissard, "Gap Labeling Theorems for Schrödinger's Operators" in From Number Theory to Physics, pp.538-630, Les Houches March 89, Springer, J.M. Luck, P. Moussa \& M. Waldschmidt Eds., (1993).

[3] J. Bellissard, "Noncommutative geometry and quantum Hall effect." in Proceedings of the International Congress of Mathematicians, Vol. 1, 2 (Zürich, 1994), 1238-1246, Birkhüser, Basel, (1995).

[4] J. Bellissard, A. van Elst, H. Schulz-Baldes, "The Non Commutative Geometry of the Quantum Hall Effect", J. Math. Phys., 35, (1994), 5373-5471. 
[5] J. Bellissard, "The Noncommutative Geometry of Aperiodic Solids", in Geometric and Topological Methods for Quantum Field Theory, (Villa de Leyva, 2001), pp. 86156, World Sci. Publishing, River Edge, NJ, (2003).

[6] J. Bellissard, P. D. Hislop, "The behavior of the current-current, and higher-order, correlation functions in the strong localization regime", in preparation.

[7] A. Bovier, M. Campanino, A. Klein, F. Perez, "Smoothness of the density of states in the Anderson model at high disorder", Commun. Math. Phys., 114, (1988), 439-461.

[8] R. Carmona, J. Lacroix, Spectral theory of random Schrödinger operators. Probability and its Applications. Birkhäuser Boston, Inc., Boston, MA (1990).

[9] A. Connes, Noncommutative Geometry. Academic Press, San Diego, CA (1994).

[10] Constantinescu, J. Fröhlich, T. Spencer, "Analyticity of the density of states and replica method for random Schrödinger operators on a lattice", J. Statist. Phys., 34, (1984), no. 3-4, 571-596.

[11] A. Figotin, L. Pastur, "An exactly solvable model of a multidimensional incommensurate structure", Commun. Math. Phys., 95, (1984), no. 4, 401-425.

[12] J. Fröhlich, T. Spencer, "A rigorous approach to Anderson localization", in Common trends in particle and condensed matter physic (Les Houches, 1983). Phys. Rep., 103, (1984), 9-25.

[13] A. Klein, O. Lenoble, P. Müller, "On Mott's formula for the ac-conductivity in the Anderson model", to appear in Ann. Math.

[14] W. Kirsch, O. Lenoble, L. Pastur, "On the Mott formula for the ac conductivity and binary correlators in the strong localization regime of disordered systems". J. Phys. A, 36, (2003), 12157-12180.

[15] N. F. Mott, J. Non-Crystal. Solids 1, 1 (1968). See also N. F. Mott, Metal-Insulator Transitions (Taylor and Francis, London, 1974).

[16] L. Pastur, "On a heuristic expansion method in the strong localization regime of the theory of disordered systems", Applied and industrial mathematics, Venice-2, 1998, 173-185, Kluwer Acad. Publ., Dordrecht, (2000).

[17] L. Pastur, A. Figotin, Spectra of Random and Almost-Periodic Operators, SpringerVerlag, (1992).

[18] S, Sakai, $C^{*}$-algebras and $W^{*}$-algebras, Ergebnisse der Mathematik und ihrer Grenzgebiete, Band 60. Springer-Verlag, New York-Heidelberg, (1971). 
[19] B.I. Shklovskii \& A.L. Efros, Electronic Properties of Doped Semiconductors, Springer, (1984).

[20] M. Shubin, "The spectral theory and the index of elliptic operators with almost periodic coefficients", Russ. Math. Surv., 34, (1979), 109-157. 